\title{
Personality traits and health-related quality of life in patients with mood and anxiety disorders
}

\author{
Annemieke van Straten ${ }^{1,2}$, Pim Cuijpers ${ }^{1,2}$, Florence J. van Zuuren ${ }^{1}$, Niels Smits ${ }^{1}$ \& Marianne Donker ${ }^{3}$ \\ ${ }^{1}$ Department of Clinical Psychology, Faculty FPP, Vrije Universiteit, Van der Boechorststraat 1, 1081 BT, \\ Amsterdam, The Netherlands (E-mail: a.van.straten@psy.vu.nl); ${ }^{2}$ Trimbos-Institute, P.O.Box 725,3500 AS, \\ Utrecht, The Netherlands; ${ }^{3}$ Department of Public Health, Erasmus MC University Medical Centre \\ Rotterdam, Rotterdam, The Netherlands
}

Accepted in revised form 1 September 2006

\begin{abstract}
Background: Health-related quality of life (HRQL) is an accepted outcome measure in patients with mood and anxiety disorders. Yet, surprisingly little attention has been paid to the determinants. In this paper we test the hypothesis that it is associated with personality traits while controlling for mental disorders. Methods: A large sample of outpatients $(\mathrm{n}=640)$ with mood and anxiety disorders was studied. The empirically supported five factor model of normal personality traits was assessed using the NEO-FFI and includes: neuroticism, extraversion, openness to experience, agreeableness, and conscientiousness. Mental disorders were assessed with the CIDI, and HRQL with the SF-36. Results: Regression analyses revealed that the NEO-FFI scores, with the exception of conscientiousness, were significantly associated with SF-36 subscales and summary scores, independently from the mental disorders. The percentage of explained variance due to the personality traits was highest for the subscales Vitality (10.0\%), Mental Health (13.3\%) and the Mental Health Summary Score (9.5\%). Furthermore, specific personality traits were related to specific SF-36 subscales. Conclusions: A low HRQL of patients with mood or anxiety disorders is not only determined by the disease or the current health but is also shaped by personality traits that are relatively stable throughout an individual's life time.
\end{abstract}

Key words: Anxiety disorders, Depressive disorder, Health-related quality of life, Personality

\section{Introduction}

Health-related quality of life (HRQL) indices reflect the patient's burden associated with disease [1]. For a number of reasons, HRQL has gained popularity as an outcome measure in both clinical practice and research, for both somatic and mental conditions. Firstly, it measures the impact of the condition on a wide range of physical, social and emotional aspects that are highly relevant to the functioning of individuals. Secondly, while a diagnosis may only be absent or present, HRQL measures may detect (small) improvements or deteriorations. Thirdly, HRQL measures describe the impact of more than one condition simultaneously. This is especially important in conditions with a high prevalence of co-morbidity, as is the case in mood disorders which often occur simultaneously with anxiety disorders.

HRQL is greatly affected by the presence of mood and anxiety disorders [2-6]. Because these disorders are also highly prevalent, they are the major cause of HRQL deficits on a population level [7, 8]. Moreover, it has been demonstrated that patients without a fully-fledged mood 
disorder (patients with minor or subsyndromal depression and those who recovered from their depressive episode) also experience substantial HRQL deficits $[9,10]$.

Yet, although two patients with the same mood or anxiety disorder may have very different HRQL scores, surprisingly little attention has been paid to determinants of HRQL in patients with common mental disorders. HRQL might be viewed as a subjective evaluation of different domains of functioning or life in general. Since personality refers to the relatively stable inclination of a person to interpret and to (re)act in a certain way in different circumstances, it is reasonable to assume that personality may play a role in determining HRQL. There are indeed some indications in the literature. Firstly, it has been demonstrated that health-related HRQL is associated with personality in healthy persons and in persons with somatic disease [11]. Secondly, it has been demonstrated [12] that HRQL is associated with personality in other mental disorders (schizophrenia and schizoaffective disorders). These authors call for an extension of these findings to other mental disorders. Thirdly, there are already some indications that personality is associated with HRQL in patients with mood disorders [13, 14]. These findings need to be confirmed since the studies used different ways of describing personality and were carried out in a non-depressed population sample [13] and a small sample of depressed patients [14].

Nowadays, personality is usually measured on the basis of the five-factor model (FFM). The five factors are: neuroticism (tendency to experience emotions in an negative way and to cope poorly), extraversion (being optimistic and having a preference for the company of other people), openness to experience (appreciation of experience for its own sake), agreeableness (orientation towards others, being altruistic), and conscientiousness (organisation, motivation, and persistence in achieving goals). They are used to distinguish between personality profiles of healthy individuals and have strong empirical support [15-19]. They do not measure personality disorders. Personality disorders are characterised by extreme scores on (a combination of) personality traits to a level that is dysfunctional for adaptation.

The aim of the present study is to test empirically the hypothesis that the various dimensions of
HRQL are associated with personality traits. Moreover, we test the hypothesis that these associations exist independently of the mood and anxiety disorders.

\section{Methods}

\section{Study design and subjects}

In this study, we conducted secondary analyses on a sample of patients participating in a randomised trial examining the effects of three types of psychological treatments (cognitive behavioural therapy, brief therapy and care as usual) for patients with mood- and anxiety disorders. The methods of the study are described in detail elsewhere [20]. In brief, subjects were recruited at seven regionally organised outpatient mental health centres (MHCs) in the Netherlands (both rural and urban regions). Therapy was offered at 12 different locations throughout these seven regions. Patients were enrolled in two steps. First, the participating MHCs screened all patients (18-65 years) who presented themselves during the inclusion period (February 2000-October 2001). Exclusion criteria were: psychotic disorder, bipolar disorder, cognitive impairment, drug dependence, poor command of the Dutch language, or high suicide risk. Inclusion criteria were: positive screen for a mood and/or anxiety disorder according to the INSTEL screen which is a Dutch modified version of the Goldberg-screen [21, 22], eligible for outpatient mental health care, and not treated in the past year by the same MHC. Second, the remaining patients were interviewed at home (baseline assessment) by trained research assistants in order to assess psychiatric diagnoses according to the DSM-IV. Patients with the following DSM-IV diagnoses were included: major depressive disorder (single episode or recurrent), dysthymic disorder, panic disorder (with or without agoraphobia), social phobia, or generalised anxiety disorder, including co-morbid diagnoses. All eligible patients were asked to participate in the study. After a full explanation of the study, 702 patients gave written informed consent. These 702 patients were randomised to one of the three treatments and subsequent interviews were held every three months until 18 months after inclusion. 


\section{Measures}

Mood and anxiety disorders were assessed using the Composite International Diagnostic Interview $[23,24]$. The CIDI is a reliable and valid standardised diagnostic interview for the assessment of mental disorders according to DSM-IV criteria by trained lay interviewers [25, 26].

Personality characteristics were assessed with the NEO-Five Factor Inventory [19, 27]. The NEO-FFI consists of 12 items for each of the five traits. For each statement, the respondent should indicate on a five-point scale how much this applies to him- or herself. Each of the five traits scores is transformed to a scale ranging from 1-9 ('standard nine'). A score of five represents the mean of the general population. The scores on the NEO-FFI result in a five factor profile of the personality of the subject.

HRQL was measured using the Dutch version of the SF-36 [28, 29], a generic self-report measure covering eight subscales (see Table 2). Each scale represents a score ranging from 0 (poor HRQL) to 100 (optimal HRQL). Furthermore, the scores on the subscales may be summarised into a physical (PCS) and mental (MCS) component summary score. These summary scores are transformed in such a way that 50 represents the mean score of the population [30]. The SF-36 has been promoted as the standard HRQL instrument in mental health research [4]. The Dutch version has good psychometric properties [29].

\section{Statistical analyses}

The scores of the NEO-FFI and the SF-36 were compared to the population norms with onesample $t$-tests. In order to explore the relationship between the personality traits and the different HRQL dimensions we used hierarchical multiple linear regression analyses. A total of 10 regression analyses were performed, each with a HRQL subscale or a HRQL summary score as the dependent variable. Each of the 10 regression models was built in three steps. During the first step we entered demographics; during the second we added the DSM-IV diagnoses next to the demographics; and in the third and final step we added the five NEO-FFI scores next to the demographics and DSM-IV diagnoses. We considered a model as improved where the percentage of explained variance $\left(\mathrm{R}^{2}\right)$ was raised significantly $(p<0.05)$ from one step to the next. Cook Distances were calculated for each regression model to check for outliers. Since all Cooks Ds were low (between 0.002 and 0.014 ) we conclude that there is no reason to exclude patients.

\section{Results}

\section{Characteristics of the patients}

During the inclusion period 5219 new patients presented themselves at the participating MHCs. During the first intake session 3611 patients turned out to be ineligible for participation in the study (because there was no indication of a mood or anxiety disorder or they fulfilled an exclusion criterion) or they refused to participate. The remaining 1608 patients were interviewed at home. Another 516 patients were not eligible since they did not meet the DSM-IV criteria of a mood or anxiety disorder. The remaining 1092 patients were asked to participate in the study and 702 patients gave informed consent. Out of these 702 patients, 640 filled out the NEO-FFI at baseline and these patients are used in the current paper. There were no statistically significant differences with regard to the number of DSM-IV diagnoses at baseline, or on the HRQL scores, between the 640 patients who did fill out the NEO-FFI and the 62 patients who did not.

Of the 640 patients studied, most were female $(61.3 \%)$ and most had a paid job $(66.1 \%$; Table 1$)$. Of this group, $298(46.6 \%)$ had one disorder only, $216(33.8 \%)$ had two, while the $126(19.7 \%)$ remaining patients had three $(\mathrm{n}=94 ; 14.7 \%)$, four $(\mathrm{n}=31 ; 4.8 \%)$ or five $(\mathrm{n}=1 ; 0.2 \%)$ disorders. Almost half of the patients (47\%) suffered from one or more mood disorders, $12 \%$ suffered from one or more anxiety disorders while the remaining $41 \%$ suffered simultaneously from mood and anxiety disorders.

The mean scores on the NEO-FFI scales were all significantly different from the mean scale scores of the general population with the exception of the Openness scale. The patients in our study scored higher on Neuroticism $(p<0.01)$ and lower on Extraversion $(p<0.01), \quad$ Agreeableness $(p<0.01)$ and Conscientiousness $(p<0.01)$. The 
Table 1. Characteristics of outpatients with mood and anxiety disorders $(n=640)$

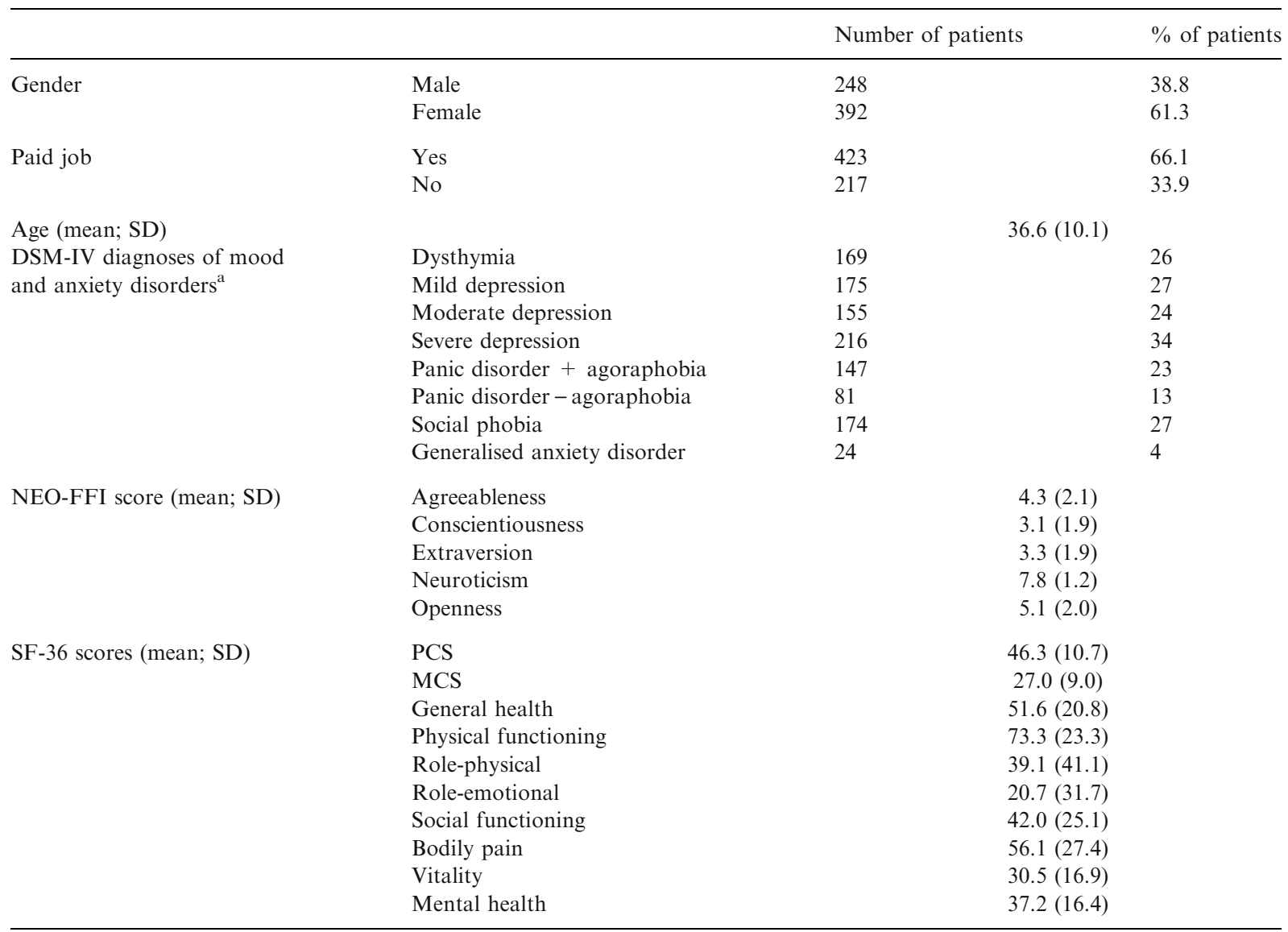

Abbreviations: PCS: physical component summary score; MCS: mental component summary score.

apatients may have more than one diagnosis.

reliabilities of the scales were sufficient to good (Agreeableness: Cronbach's $\alpha=0.71$; Conscientiousness $\alpha=0.75$; Extraversion $\alpha=0.78$; Neuroticism $\alpha=0.80$; Openness $\alpha=0.69$ ).

The mental health of the studied patients, as measured with the SF-36, was considerably worse (mean mental component summary score 27.0) than the average mental health of the population (mean mental component summary score $50 ; p<0.01)$. In contrast, the physical health (mean physical component summary score 46.3) was nearly equivalent (mean physical component summary score $50 ; p=0.64$ ).

\section{Personality traits and HRQL scores}

Table 2 shows the statistically significant results of the linear regression analyses for each HRQL subscale and both summary scores. Each regression model was built in three steps. The demographic variables, which were entered first in the regression analyses, do not explain much of the variance of the HRQL scores. The subscale physical functioning is an exception with $8 \%$ explained variance. All regression models showed a statistically significant increase in explained variance after adding the DSM-IV diagnoses (step 2). The largest improvements were found for the subscale Mental Health (16.4\% improvement), the MCS (12.7\% improvement) and the subscale Social Functioning (11.3\% improvement). Adding the five personality traits to the regression models (the third and final step) further increased the explained variance for all subscales (with the exception of Role-Physical) and both summary scores. The largest improvements were found for the subscale Mental Health 
Table 2. The association between the big five personality traits on the one hand and the different HRQL dimensions on the other while controlling for demographics and different DSM-IV diagnosis (beta coefficients and their 95\% confidence intervals of the statistically significant $(p<0.05)$ associations)*

\begin{tabular}{|c|c|c|c|c|c|}
\hline & General health & Physical functioning & Role-physical & Role-emotional & Social functioning \\
\hline STEP $1 R^{2}$ & $1.2 \%$ & $8.0 \%$ & $2.3 \%$ & $0.7 \%$ & $1.1 \%$ \\
\hline $\operatorname{STEP} 2 \mathrm{R}^{2}\left(\mathrm{R}^{2}\right.$ CHANGE $)$ & $6.7 \%(5.5 \%)^{*}$ & $14.7 \% \mathrm{~s}(6.7 \%)^{*}$ & $5.6 \%(3.3)^{*}$ & $7.4 \%(6.7 \%)^{*}$ & $12.4 \%(11.3 \%)^{*}$ \\
\hline STEP $3 R^{2}$ ( $R^{2}$ CHANGE): & $15.6 \%(8.8 \%)^{*}$ & $17.5 \%(2.8 \%)^{*}$ & $6.6 \%(1.1)$ & $11.2 \%(3.8 \%)^{*}$ & $16.8 \%(4.4 \%)^{*}$ \\
\hline Age & & $-0.4(-0.6 /-0.2)$ & $-0.6(-0.9 /-0.3)$ & & \\
\hline Female & & $-6.3(-9.9 /-2.6)$ & & & \\
\hline No paid job & & $-4.2(-7.9 /-0.5)$ & & $5.4(0.2 / 10.6)$ & \\
\hline \multicolumn{6}{|l|}{ DSM-IV diagnoses } \\
\hline Dysthymia & & & & & $-4.4(-8.7 /-0.1)$ \\
\hline Depression mild & & & & $-11.5(-19.6 /-3.5)$ & $-8.7(-14.8 /-2.6)$ \\
\hline Depression moderate & $-8.0(-13.2 /-2.7)$ & $-6.1(-11.9 /-0.3)$ & $-16.2(-27.1 /-5.3)$ & $-17.7(-25.8 /-9.5)$ & $-11.8(-18.1 /-5.6)$ \\
\hline Depression severe & $-7.0(-11.9 /-2.1)$ & $-10.7(-16.1 /-5.2)$ & $-19.5(-29.8 /-9.3)$ & $-21.6(-29.3 /-13.9)$ & $-16.4(-22.3 /-10.6)$ \\
\hline Panic d. + agoraphobia & $-4.8(-8.7 /-0.9)$ & $-6.4(-10.7 /-2.1)$ & & & $-8.6(-13.2 /-3.9)$ \\
\hline \multicolumn{6}{|c|}{ Panic d. - agoraphobia } \\
\hline Social phobia & & & & & $-4.8(-9.1 /-0.5)$ \\
\hline \multicolumn{6}{|l|}{ GAD } \\
\hline \multicolumn{6}{|l|}{ NEO-FFI } \\
\hline Agreeableness & $1.1(0.3 / 1.9)$ & $1.1(0.2 / 1.9)$ & $2.1(0.5 / 3.7)$ & & $1.9(1.0 / 2.8)$ \\
\hline \multicolumn{6}{|l|}{ Conscientiousness } \\
\hline Extraversion & $1.3(0.3 / 2.3)$ & & & & \\
\hline Neuroticism & $-2.8(-4.3 /-1.4)$ & & & $-3.5(-5.8 /-1.2)$ & \\
\hline \multirow[t]{2}{*}{ Openness } & & & & $-2.0(-3.2 /-0.7)$ & $-1.4(-2.3 /-0.4)$ \\
\hline & Bodily pain & Vitality & Mental health & PCS & MCS \\
\hline STEP $1 R^{2}$ & $0.9 \%$ & $2.6 \%$ & $1.1 \%$ & $3.6 \%$ & 0.6 \\
\hline STEP $2 R^{2}$ ( $R^{2}$ CHANGE) & $5.1 \%(4.2 \%)^{*}$ & $12.5 \%(9.9 \%)^{*}$ & $17.5 \%(16.4 \%)^{*}$ & $6.8 \%(3.3 \%)^{*}$ & $13.3 \%(12.7 \%)^{*}$ \\
\hline STEP $3 R^{2}$ ( $R^{2}$ CHANGE): & $7.6 \%(2.5 \%)^{*}$ & $22.5 \%(10.0 \%)^{*}$ & $30.8 \%(13.3 \%)^{*}$ & $9.5 \%(2.6 \%)^{*}$ & $22.8 \%(9.5 \%)^{*}$ \\
\hline Age & & & & $-0.1(-0.2 /-0.1)$ & \\
\hline Female & & $-4.5(-7.0 /-1.9)$ & & & \\
\hline No paid job & & $3.0(0.4 / 5.6)$ & & & $1.4(0.1 / 2.8)$ \\
\hline \multicolumn{6}{|l|}{ DSM-IV diagnoses } \\
\hline \multicolumn{6}{|l|}{ Dysthymia } \\
\hline Depression mild & & $-8.3(-12.2-4.3)$ & $-8.9(-12.5 /-5.2)$ & & $-5.0\left(-7.1^{/}-2.8\right)$ \\
\hline Depression moderate & & $-12.0(-16.0-7.9)$ & $-12.0\left(-15.7^{\prime}-8.2\right)$ & & $-6.8(-9.0-4.6)$ \\
\hline Depression severe & & $-11.9\left(-15.7^{/}-8.1\right)$ & $-13.2(-16.7 /-9.6)$ & $-3.0(-5.6-0.4)$ & $-7.6(-9.6-5.5)$ \\
\hline Panic d. + agoraphobia & $-6.2(-11.6-0.9)$ & & $-6.8(-9.6-4.0)$ & & $-2.0(-3.6-0.3)$ \\
\hline \multicolumn{6}{|c|}{ Panic d. - agoraphobia } \\
\hline \multicolumn{6}{|l|}{ Social phobia } \\
\hline \multicolumn{6}{|l|}{ GAD } \\
\hline \multicolumn{6}{|l|}{ NEO-FFI } \\
\hline Agreeableness & $1.8(0.7 / 2.8)$ & & & $0.7\left(0.3^{\prime} 1.1\right)$ & \\
\hline \multicolumn{6}{|l|}{ Conscientiousness } \\
\hline Extraversion & & $2.4\left(1.7^{/ 3} 3.2\right)$ & $1.2\left(0.5^{\prime} 1.8\right)$ & & $0.5\left(0.1^{/} 0.9\right)$ \\
\hline Neuroticism & & $-1.5(-2.6-0.4)$ & $-4.4(-5.5 /-3.4)$ & & $-1.8(-2.4-1.2)$ \\
\hline Openness & & $-1.0\left(-1.7^{\prime}-0.4\right)$ & & & $-0.8\left(-1.1^{/}-0.4\right)$ \\
\hline
\end{tabular}

${ }^{*} p<0.05$; step 1: only demographics were entered; step 2: DSM-IV diagnoses were added next to the demographics; model 3: personality traits were added next to the demographics and the DSM-IV diagnoses.

Abbreviations: GAD: Generalised Anxiety Disorder; PCS: physical component summary score; MCS: mental component summary score. 
(13.3\% improvement), the subscale Vitality (10.0\% improvement) and the MCS (9.5\% improvement). These three scales also showed the highest percentages of explained variance $(30.8 \%, 22.5 \%$ and $22.8 \%$ respectively).

Older age was significantly associated with poorer (role- and overall) Physical Functioning, being female with poorer Physical Functioning and Vitality, while having no paid job was associated with poorer Physical Functioning and a higher Role-Emotional, Vitality and MCS score.

Furthermore, there were significant associations between the different DSM-IV diagnoses and the HRQL subscales and summary scores. Generalised anxiety disorder and panic disorder without agoraphobia were the only two diagnoses which were unrelated to the HRQL dimensions. Dysthymia and social phobia were each related to one subscale (Social Functioning) only. Depression (mild, moderate, severe) and panic disorder with agoraphobia were related to many different subscales and summary scores.

Most importantly, there were also significant associations between the different personality traits and the HRQL subscales and summary scores, independent of the demographics and DSM-diagnoses just mentioned. Conscientiousness (organisation, motivation and persistence in achieving goals) was the only personality trait unrelated to HRQL. Agreeableness, which refers to the extent of altruistic or antagonistic orientation towards others, is primarily associated with physical subscales. It is the only personality trait that is associated with the PCS. The association is positive, which means that a higher score on the agreeableness subscale is associated with better physical health. All three remaining personality traits (Neuroticism, Openness and Extraversion) are associated with the Vitality subscale and with the MCS. In addition, Neuroticism (the tendency to experience negative emotions and cope poorly) and Extraversion (the quantity and intensity of interpersonal interactions and positive emotions) are associated with General Health and Mental Health. All associations with Neuroticism are negative which means that a lower degree of Neuroticism corresponds with a higher HRQL score. All associations with Extraversion are positive. Finally, Openness which may be described as the appreciation of experience for its own sake is associated with Role-Emotional, and Social Functioning. Surprisingly, all associations are negative, which means that a lower score for openness corresponds with higher HRQL scores.

\section{Discussion}

Our results confirm the well-known association between mood and anxiety disorders and HRQL. The main contribution of this paper is that we demonstrated that personality traits are associated with different dimensions of HRQL independently of the presence of mood and/or anxiety disorders. Agreeableness was related to the Physical Component Summary score, while Neuroticism, Extraversion and Openness were related to the Mental Component Summary score.

Our study has several strengths. First of all, the number of patients included in this study was relatively large. Furthermore, all subjects were examined with extensive standardised diagnostic interviews, and all patients had established mood and/or anxiety disorders. However, the studied patients may not be representative with regard to the personality traits since they were all willing to participate and to be extensively interviewed. Another limitation of the study is that personality characteristics were assessed only once. Although personality characteristics are generally regarded as traits that are stable over time, it has been demonstrated that changes do occur in the long term [31]. The cross-sectional design prevents us from examining possible changes and their relationship to the changes in HRQL and the occurrence of mood- and anxiety disorders.

We demonstrated that different personality traits were associated with different HRQL dimensions. The association between Neuroticism and Extraversion with HRQL and subjective wellbeing has been demonstrated before in patients with a somatic condition and in healthy subjects $[11,32]$. It is remarkable that persons with a high Agreeableness score have a better HRQL with regard to social and physical aspects but not emotional aspects. Possibly, persons with a high level of Agreeableness have more friends, are more relaxed and pay less attention to physical 
discomfort or pain. As far as we know, this has not been found in earlier studies, and replication is necessary before any definite conclusion can be drawn. We also found Extraversion to be positively associated with several aspects of HRQL. The focus on external contacts associated with extraversion may also take away the focus on one's own problems and thus result in a higher HRQL. But again, there are no earlier studies which confirm our findings, and more research in this area is necessary.

It is well established that scores on personality traits of patients with mental disorder differ significantly from the scores of other persons [33-35]. In particular, Neuroticism has been shown to be strongly related to the presence of mental disorder $[33,34]$. However, the nature of this association is still a highly debated issue [36]. The deviant scores on personality traits may be one of the causes of (repeated) episodes of illness, or one of the consequences, but there might also exist a continuum with the personality trait as an attenuated form of the mental disorder. The most interesting finding of this paper is however that we demonstrated that Neuroticism is associated with HRQL independently of the mental disorders.

In conclusion we found that personality traits are significantly associated with HRQL scores, independent of the relationship of HRQL with demographic variables and DSM-IV mood and anxiety disorders. This indicates that HRQL is not only influenced by the disease, current health, or current situation, but is also shaped by personality traits that are relatively stable throughout an individual's lifetime.

\section{Acknowledgement}

The study was funded by The Netherlands Organisation for Health Research and Development (ZonMw; project 9605011)

\section{References}

1. Aaronson NK. Quality of life: what is it? How should it be measured? Oncology 1988; 2: 69-74.

2. Bijl RV, Ravelli A. Current and residual functional disability associated with psychopathology: Findings from the Netherlands Mental Health Survey and Incidence Study (NEMESIS). Psychol Med 2000; 30: 57-668.
3. Kruijshaar ME, Hoeymans N, Bijl RV, Spijker J, EssinkBot ML. Levels of disability in major depression. Findings from the Netherlands Mental Health Survey and Incidence Study (NEMESIS). J Affect Disord 2003; 77: 53-64.

4. Mendlowicz MV, Stein MB. Quality of life in individuals with anxiety disorder. Am J Psychiatry 2000; 157: 669-682.

5. Pirraglia PA, Rosen AB, Hermann RC, Olchanski NV, Neumann P. Cost-utility analysis Studies of depression management: a systematic review. Am J Psychiatry 2004; 161: 2155-2162.

6. Weel-Baumgarten EMVan, Schers HJ, Bosch WJVan den, Hoogen HJVan den, Zitman FG. Long-term follow-up of depression among patients in the community and in family practice settings. A systematic review. J Fam Pract 2000; 49: $1113-1120$.

7. WHO World Health Organization. World Health Report 2003; Shaping the future. Geneva: World Health Organization, 2003.

8. Melse JM, Essink-Bot ML, Kramers PGN, Hoeymans N. A national burden of disease calculation: Dutch disability-adjusted life-years. Am J Public Health 2000; 90: 1241-1247.

9. Cuijpers P, Graaf Rde, Dorsselaer Svan. Minor depression: Risk profiles, functional disability, health care use and risk of developing major depression. J Affect Disord 2004; 79: 71-79.

10. Rapaport MH, Judd LL. Minor depressive disorder and subsyndromal depressive symptoms: functional impairment and response to treatment. J Affect Disord 1998; 48: 227-232.

11. Yamaoka K, Shigehisa T, Ogoshi K, Watanabe M, Hayashi F, Hayashi C. Health-related quality of life varies with personality types: a comparison among cancer patients, non-cancer patients and healthy individuals in a Japanese population. Qual Life Res 1998; 7 : 535-544.

12. Kentros MK, Terkelsen K, Hull J, Smith TE, Goodman M. The relationship between personality and quality of life in persons with schizoaffective disorder and schizophrenia. Qual Life Res 1997; 6: 118-122.

13. Grucza RA, Przybeck TR, Spitznagel EL, Cloninger CR. Personality and depressive symptoms: A multi-dimensional analysis. J Affect Disord 2003; 74: 123-130.

14. Kuehner C, Buerger C. Determinants of subjective quality of life in depressed patients: The role of self-esteem, response styles, and social support. J Affect Disord 2005; 86: 205-213.

15. Bienvenue OJ, Brown C, Samuels JF, Liang KY, Costa PT, Eaton WW, Nestadt G. Normal personality traits and comorbidity among phobic, panic and major depressive disorders. Psychiatry Res 2001; 102: 73-85.

16. John OP. Towards a taxanomy of personality descriptors. In: Buss DM, Cantor N (eds), Personality Psychology: Recent Trends and Emerging Directions. New York: Springer Publishing, 1989: 261-271.

17. Goldberg LR. The structure of phenotypic personality traits. Am Psychol 1993; 48: 26-34.

18. Trull TJ, Sher KJ. Relationship between the five-factor model of personality traits and axis I disorders in a nonclinical sample. J Abnorm Psychol 1994; 103: 350-360. 
19. Costa PT, McCrae RR. Revised NEO Personality Inventory (NEO-PR-I), the Five Factor Inventory (NEO-FFI): Professional Manual. Psychological Assessment Resources. Odessa: FL, 1992.

20. Straten AVan, Tiemens B, Hakkaart L, Nolen WA, Donker M. Stepped care versus matched care for mood and anxiety disorders: A pragmatic randomised trial. Acta Psychiatr Scand 2006; 113: 468-476.

21. Goldberg D, Bridges K, Duncan-Jones P, Grayson D. Detecting anxiety and depression in general medical settings. Br Med J 1988; 297: 897-899.

22. Tiemens BG. Management of mental health problems in primary care. The doctor, the patient and the medical model. Groningen: Ponsen \& Looijen, 1999.

23. WHO World Health Organisation. Composite International Diagnostic Interview (CIDI), version 2.1, 12 months. Geneva: World Health Organisation, 1997.

24. Ter Smitten MH, Smeets RMW, Brink WVan den. Composite International Diagnostic Interview (CIDI) [in Dutch], version 2.1, 12 months. Amsterdam: World Health Organisation, 1998.

25. Andrews G, Peters L. The psychometric properties of the Composite International Diagnostic Interview. Soc Psychiatry Psychiatr Epidemiol 1998; 33: 80-88.

26. Wittchen H-U. Reliability and validity studies of the WHO- Composite International Diagnostic Interview (CIDI): A critical review. J Psychiatry Res 1994; 8: 57-84.

27. Hoekstra HA, Ormel J, De Fruyt F. De NEO-PI-R/NEOFFI; Big Five persoonlijkheidsvragenlijsten; Handleiding. [Manual of the Dutch version of the NEO-PR-I/NEOFFI]. Lisse: Swets and Zeitlinger, 1996.

28. Van der Zee K, Sanderman R. Assessment of General Health with the RAND36. Manual (in Dutch). Groningen:Noordelijk Centrum voor Gezondheidsvraagstukken/ Rijksuniversiteit Groningen, 1993.
29. Ware JE Jr, Sherbourne CD. The MOS 36-item Short Form Health Survey (SF36) I. Conceptual framework and item selection. Med Care 1992; 30: 473-483.

30. Ware JE. SF-36 health survey update. Spine 2000; 25 : 3130-3139.

31. Roberts BW, Walton KE, Viechtbauer W. Patterns of mean-level change in personality traits across the life course: A meta-analysis of longitudinal studies. Psychol Bulletin 2006; 132: 1-25.

32. Schimmack U, Oishi S, Furr RM, Funder DC. Personality and life satisfaction: a facet-level analysis. Pers Soc Psychol Bull 2004; 30: 1062-1075.

33. Oldehinkel AJ, Bouhuys AL, Brilman E, Ormel J. Functional disability and neuroticism as predictors of late-life depression. Am J Geriat Psychiatry 2001; 9: 241-248.

34. Ormel J, Wolfarth T. How neuroticism, long-term difficulties, and life situation change influence psychological distress: A longitudinal model. J Pers Soc Psychol 1991; 60: 744-755.

35. Petersen T, Bottonari K, Alpert JE, Fava M, Nierenberg AA. Use of the five-factor inventory in characterising patients with major depressive disorder. Compr Psychiatry 2001; 42: 488-493.

36. Sass H, Jünemann K. Affective disorders, personality and personality disorders. Acta Psychiatr Scand 2003; 108: 34 40.

Address for Correspondence: Annemieke van Straten, Department of Clinical Psychology, Faculty FPP, Vrije Universiteit, Van der Boechorststraat 1, 1081 BT, Amsterdam,

The Netherlands

Phone: + 31-20-5988970; Fax: + 31-20-5988758

E-mail: a.van.straten@psy.vu.nl 